\title{
Oritavancin for the treatment of complicated gram-positive infection in persons who inject drugs
}

\author{
Aileen Ahiskali ${ }^{1}$ and Heather Rhodes ${ }^{2^{*}}$ (D)
}

\begin{abstract}
Background: Treatment of complicated infections in persons who inject drugs (PWID) and patients experiencing homelessness poses a unique challenge to clinicians. Long-acting lipoglycopeptide antibiotics, such as oritavancin, may facilitate extended courses of outpatient intravenous therapy while avoiding the need for central lines, improving compliance and thus increasing the chance of clinical cure.

Methods: Retrospective chart review of adult PWID who received at least one dose of oritavancin for a gram-positive infection between 1/1/17 and 6/30/19 at a large safety net hospital.

Results: Twenty three PWID received 24 courses of at least one dose of oritavancin for a gram-positive infection; 16 were experiencing homelessness at the time of diagnosis. Methicillin resistant Staphylococcus aureus (MRSA) was the most common infecting pathogen and bone or joint the most frequent infection site. Nineteen encounters resulted in clinical cure, including 5 whose conditions improved despite non-adherence to their prescribed regimen. Three patients experienced a non-favorable outcome. Two patients experienced mild adverse drug reactions that did not interfere with therapy; no patients died while on therapy.

Conclusion: Oritavancin may be a clinically effective treatment option for the management of complicated gram-positive infections in PWID and patients experiencing homelessness. Further studies should be performed to validate these results.
\end{abstract}

\section{Introduction}

Effective strategies to improve serious infection treatment outcomes in non-adherent patients are lacking and complicated by injection drug use (IDU) and homelessness. Up to $70 \%$ of persons who inject drugs (PWID) experience at least 1 bacterial skin infection in their lifetime, but it is difficult to accurately describe the true breadth of the problem as a recent study found that more than half of all IDUassociated bacterial infections (ABIs) may be

\footnotetext{
* Correspondence: heather.rhodes@hcmed.org

${ }^{2}$ Clinical Quality Improvement, Hennepin Healthcare, Orange 1.220-7, 701 Park Avenue, Minneapolis, MN 55415, USA

Full list of author information is available at the end of the article
}

unrecorded [1-4]. Adding further complexity to treatment, the prevalence of homelessness among PWID has been reported to be as high as 59\% [5]. Homelessness significantly increases the risk of relapse in those who have previously stopped injecting, promoting initial and recurrent IDU-ABIs [6].

Complex socioeconomic factors often limit the ability to administer first-line therapies. Outpatient parenteral antibiotic therapy (OPAT) is controversial due to concerns of inappropriate central line access and treatment failure risk [7]. Eaton et al. challenged this apprehension by employing a 9-point risk assessment to successfully administer OPAT to PWID, reducing average length of stay from 42 to 22 days [8].

(c) The Author(s). 2020 Open Access This article is licensed under a Creative Commons Attribution 4.0 International License, which permits use, sharing, adaptation, distribution and reproduction in any medium or format, as long as you give appropriate credit to the original author(s) and the source, provide a link to the Creative Commons licence, and indicate if changes were made. The images or other third party material in this article are included in the article's Creative Commons licence, unless indicated otherwise in a credit line to the material. If material is not included in the article's Creative Commons licence and your intended use is not permitted by statutory regulation or exceeds the permitted use, you will need to obtain permission directly from the copyright holder. To view a copy of this licence, visit http://creativecommons.org/licenses/by/4.0/ The Creative Commons Public Domain Dedication waiver (http://creativecommons.org/publicdomain/zero/1.0/) applies to the data made available in this article, unless otherwise stated in a credit line to the data. 
However, after providers form trusting relationships with patients, home infusion services may still refuse to provide therapy to patients they deem high risk. When oral antibiotics are therapeutically appropriate, drug interactions (e.g. with rifampin), cost and repeated non-adherence may preclude use of this route. Thus, at times patients must remain admitted for multiple weeks to complete therapy, or leave against medical advice (AMA) and risk the potentially lifethreatening consequences of inadequately treated infections. Additionally, prolonged admissions delay patient enrollment in outpatient rehabilitation programs, which in turn slows substance use disorder (SUD) treatment.

Oritavancin (ORI) is a long-acting lipoglycopeptide (LAL) that covers a broad range of grampositive pathogens including methicillin-resistant and methicillin susceptible Staphylococcus aureus (MRSA, MSSA), Streptococcus species, and vanAmediated vancomycin-resistant Enterococcus species and was FDA approved in 2014 for the treatment of acute bacterial skin and skin structure infections (ABSSSI) as a one-time 3 hour $1200 \mathrm{mg}$ intravenous (IV) infusion [9]. Its long terminal half-life, large volume of distribution and penetration to bone and joint spaces make it appealing for treatment of deep seated infections that require IV therapy, but in whom this route may not be feasible in the outpatient setting. At our institution, ORI was selected as the formulary LAL due to patient assistance programs that were more relevant to our patient population and a lack of evidence demonstrating clinical superiority of dalbavancin (DAL), the other LAL, over oritavancin.

Hennepin County Medical Center (HCMC) is the largest safety net hospital in the state of Minnesota and a Level 1 Trauma and Burn destination caring for patients across the upper Midwest. Providers at our 484 bed institution routinely face challenges providing IV therapy for patients afflicted with serious mental health issues, SUD and homelessness. In 2018, HCMC providers treated over 130 cases of Staphylococcus aureus bacteremia, of which approximately one-third were attributed to IDU. Additionally, during 2018 alone, at least $10 \%$ of patients were readmitted with recurrent $S$. aureus bloodstream infections primarily due to non-adherence and/or reinfection. In an effort to provide adequate treatment courses, single- or multiple-dose regimens of ORI have been employed in select patients at our institution. We describe our clinical experience utilizing ORI for the treatment of complicated gram-positive infections in adult PWID, many of whom experienced homelessness.

\section{Methods}

We performed a retrospective cohort analysis of PWID treated with ORI for gram-positive infection. Patients were included if they were 18 years of age or older and received at least one dose of ORI for the treatment of documented or presumed grampositive endocarditis, bone/joint infection, bacteremia, or skin and soft tissue infection between $1 / 1 / 17$ and 6/30/19. For patients who received more than one course of oritavancin during the study period, each course was assessed separately for inclusion. All patients in this study were evaluated by an infectious diseases (ID) physician as prescription of ORI is restricted to ID physicians at our institution due to its high drug acquisition cost and broad gram-positive spectrum of activity; pharmacy does not release ORI unless authorized by ID. Infection indication was identified by the ID consult note. Susceptibility testing for ORI was not performed on any isolates, but was inferred from vancomycin susceptibility based on previous studies' findings [10]. Patients who received concomitant antibiotics were included in the analysis; their additional therapy is included in Table 1. For multiple dose regimens, doses were administered once weekly until completed. Patients were excluded if more than 14 days elapsed between administered doses, their care was palliative in nature, they were pregnant or a prisoner at the time of treatment, or had declined use of their information for research purposes.

Both authors independently reviewed each case and made a determination of outcome. Results were categorized as clinical cure or failure based on manual chart review. Outcomes were further described as incomplete adherence/cure $(\mathrm{I} / \mathrm{C})$ or incomplete adherence/failure (I/F) if a dose was missed based on the initially planned regimen. Clinical cure was defined as resolution of signs and symptoms of infection (fever, white blood cell count, C-reactive protein) without need for additional antimicrobial therapy following completion of ORI, excluding long term suppressive antibiotics for patients with retained hardware. Failure was defined as progression of gram-positive infection and need for alternative therapy. Outcomes were reviewed out to 60 days after the final ORI infusion.

Adverse drug reactions (ADRs) were collected up to 6 weeks from the last dose or up to the point that a patient was lost to follow up. All notes available in the institution's electronic medical record (EMR) were examined for mention and description of ADRs. Outside hospital records were reviewed if available at the time of review by way of Epic's Care Everywhere. The Naranjo Adverse Drug Reaction Probability Scale (NADRPS) was 


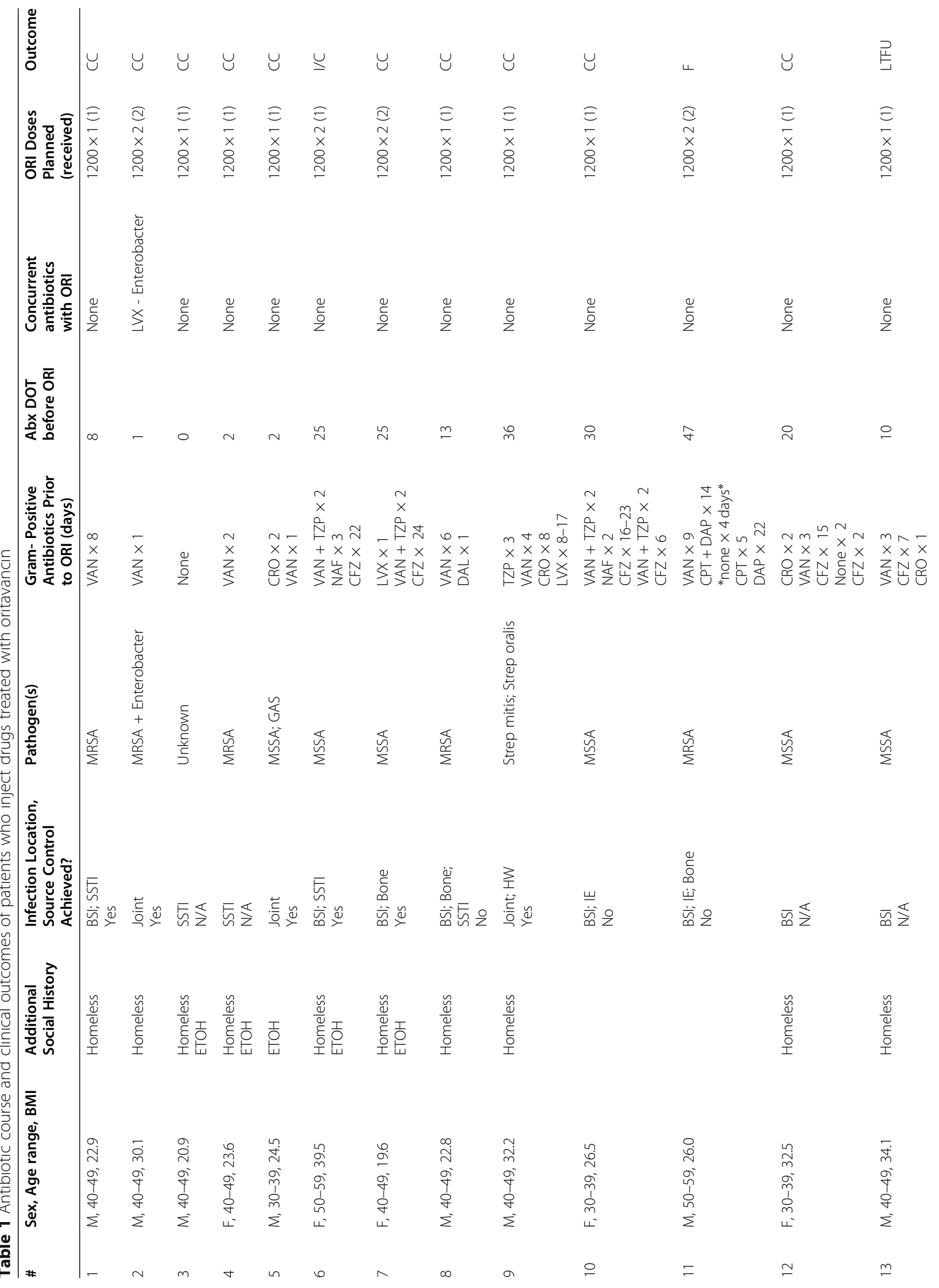




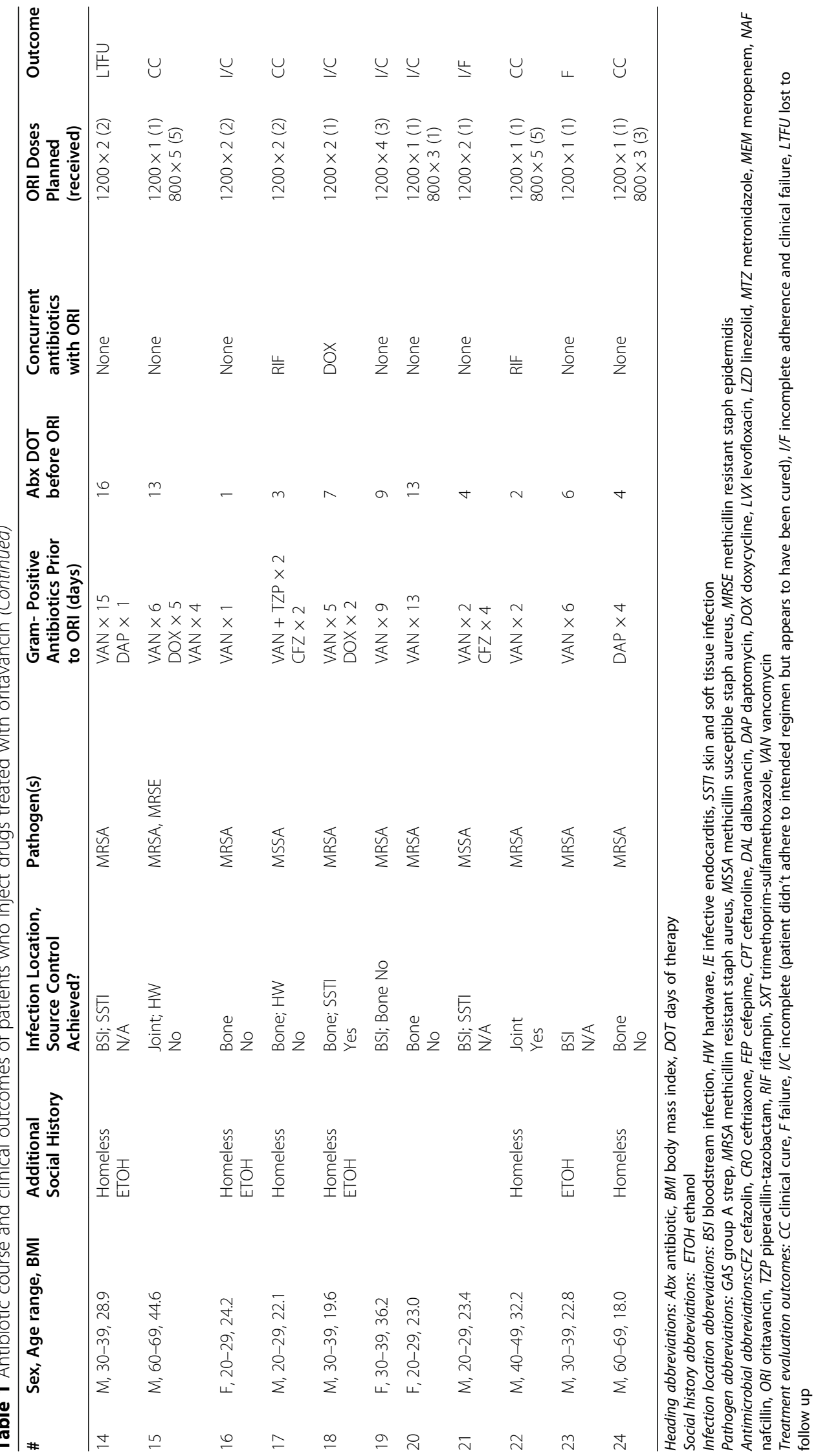


employed to determine the probability the ADR was attributable to oritavancin [11].

This study was reviewed and approved by the Human Subjects Research Committee of the Hennepin Healthcare Research Institute.

\section{Results}

A list of all 37 encounters in which a patient received one or more doses of oritavancin within the specified time frame supplied the starting point for review. Thirteen encounters were excluded; the primary reason for exclusion was no known history of injection drug use $(n=9)$; other reasons included a diagnosis outside of the inclusion criteria $(n=2)$ and greater than 14 days elapsed between administered doses $(n=2)$. Twenty four courses, prescribed to 23 different patients, were included in the analysis. One patient (numbers 2 and 22 in Table 1) received 2 separate courses of treatment, with the second course administered 20 months after the final dose of the initial course. At the time of infection diagnosis, 16/24 (67\%) encounters were for patients experiencing homelessness. Most patients were male (16/ $23,70 \%$ ) and the average age was 41 years old (range 22-64). The median body mass index (BMI) was 24.3; 8/24 (33\%) were considered obese with a BMI of greater than 30 . Sixteen of 23 patients $(70 \%)$ had a history of significant psychiatric illness defined as schizophrenia, bipolar disorder, major depressive disorder, schizoaffective disorder or borderline personality disorder. Nine (39\%) patients left AMA during a prior hospitalization for their infection.

MRSA was the most common infecting pathogen occurring in $14 / 24$ (58\%) of encounters. Bone or joint was the most frequent infection location occurring in 14/24 $(58 \%)$ encounters, and the spine was the most common site of bone infection $(4 / 9,44 \%)$. Half of all encounter patients $(12 / 24,50 \%)$ were bacteremic with either MRSA $(6 / 12,50 \%)$ or MSSA $(6 / 12,50 \%)$. Of the two patients diagnosed with infective endocarditis, both involved native tricuspid valves and neither underwent surgical valve replacement.

Patients received a median of 9.5 days of effective gram-positive therapy based on susceptibility, when available, prior to ORI initiation. Number of ORI doses ranged from 1 to 6; initial doses were universally $1200 \mathrm{mg}$. Subsequent dosing was at the discretion of the clinician and was always administered weekly. Eleven patients received more than one dose. Of those, 4 received $800 \mathrm{mg}$ doses and $7 \mathrm{re}-$ ceived $1200 \mathrm{mg}$ doses. The majority of doses were administered in the outpatient infusion center. Twelve patients received 1 dose as inpatient; 3 of these patients received additional outpatient doses thereafter. Twenty three of 24 encounters utilized ORI for therapy completion, having received prior gram-positive treatment for their infections. Patient 8 was the only patient to also receive DAL; the patient received a single dose at an outside hospital prior to transferring care to our institution.

Clinical cure was achieved in 19/24 (79\%) encounters and failure in $3 / 24(13 \%) ; 2 / 24(8 \%)$ were lost to follow-up after their last infusion. The two patients lost to follow-up were not noted to have any signs of worsening of infection at their final infusion, however, documentation was limited at each of those visits and thus no assessments can be made. Of the 4 patients with osteomyelitis involving the spine, $3 / 4$ (75\%) experienced clinical cure and $1 / 4$ (25\%) treatment failure. Of the 6 patients who had incomplete adherence to the planned regimen, cure was seen in 5 patients and failure in 1 . Three patients received only the first of two planned doses; including the patient deemed to be a clinical failure in the group with partial adherence. The remaining 3 patients with partial adherence experienced an unexpected delay of 14 days between doses, but received all planned doses (3 to 5 doses total).

Two patients (8\%) experienced ADRs within 6 weeks of receiving ORI. One patient presented to the Emergency Department (ED) 5 days after receiving ORI complaining of sharp, non-radiating abdominal pain, however, the patient eloped prior to evaluation. Four days after her ED visit she was admitted to the jail medical ward, which is overseen by our institution, without documentation of infection, pain or further antibiotics. One patient experienced an infusionrelated reaction becoming visibly flushed and complaining of a headache $20 \mathrm{~min}$ into the first dose. After an infusion pause, the remaining drug was administered without symptom recurrence and she received a second dose 1 week later without issue. Employing the NADRPS harm scale, the former patient's ADR was classified as possible and the latter as probable.

Three patients (patients 11,21, and 23) were determined to have failed therapy with ORI, with 1 of 3 possibly related to incomplete adherence. Patient 11 was being treated for MRSA ((vancomycin minimum inhibitory concentration (MIC) 2, confirmed by two methods, Vitek and Microscan)) bacteremia with vertebral osteomyelitis and was initially bacteremic for 14 consecutive days. Source control was unable to be achieved due to location of fluid collections and proximity to spinal cord. After blood culture clearance, he was discharged and returned for daily infusions of high-dose daptomycin $(>10 \mathrm{mg} / \mathrm{kg}$ ) but was transitioned to weekly ORI 3 weeks later due to loss of IV 
access and inability to receive a peripherally inserted central catheter (PICC). After the second ORI infusion, the patient was readmitted due to ongoing back pain and new spinal cultures were obtained; daptomycin MIC had increased from 0.5 to $4 \mu \mathrm{g} / \mathrm{mL}$ and thus was no longer susceptible. Although the corresponding vancomycin MIC of this isolate was $1 \mu \mathrm{g} / \mathrm{mL}$, the ID team did not treat with vancomycin due to recent history of MRSA isolate with vancomycin MIC $2 \mu \mathrm{g} /$ $\mathrm{mL}$ and the concern for emergence of resistant subpopulations. At the discretion of the ID physician, the patient remained admitted to complete 6 weeks of ceftaroline.

Patient 21 was treated for MRSA bacteremia and ABSSSI and missed the second of two planned ORI doses. He was admitted 14 days after his ORI infusion (7 days after the missed dose) and found to have recurrent MRSA bacteremia along with a new aortic valve vegetation on transthoracic echocardiography (TTE) that was not seen on TTE during his last admission.

Patient 23 is a paraplegic man with no sensation in his lower extremities, initially treated with IV vancomycin for MRSA bacteremia. On day 7 he planned to leave AMA; oral antibiotics could not be prescribed due to drug interactions so he was given a single ORI dose. The patient was readmitted for inpatient psychiatry care a few weeks later where he experienced a femur fracture while adjusting himself in bed. He was taken to surgery; intraoperative cultures grew MRSA but blood cultures remained negative. Both MRSA isolates, from the pre-ORI blood cultures and postORI intraoperative cultures, had a vancomycin MIC of $1 \mu \mathrm{g} / \mathrm{mL}$ via Vitek.

\section{Discussion}

We describe our real-world experience using ORI for the treatment of complicated gram-positive infections in PWID, many of whom were also experiencing homelessness. Despite having limited treatment options for patients that are noncompliant with oral therapies or otherwise not candidates to receive IV antibiotics, alternative therapeutic regimens are often discredited or avoided due to a lack of robust clinical evidence. Although more data are emerging regarding LAL use for complicated infections, studies specific to ORI use in vulnerable populations remain limited.

When comparing our study to others that included outcomes specific to persons who use drugs (PWUD), it is important to note that each investigation evaluated outcomes differently. In an effort to compare findings despite methodological differences, data specific to PWUD in similar studies are outlined in Table 2. Overall, our rates of clinical cure $(79 \%)$ and failure (13\%) were similar to other reports [12-16].

A comparable study focused on complicated infections in vulnerable patients treated with LALs but outcomes were specific to DAL [16]. Although ORI and DAL are similar, it is important to highlight their differences to further appreciate our study's contribution to existing literature. While both boast an exceptionally long half-life $\left(t^{1} / 2\right)$ based on population pharmacokinetic analyses, ORI's terminal $t^{1 / 2}$ is slightly longer, 10.2 vs. 8.5 days and the plasma protein binding of DAL is $93 \%$ as compared to $85 \%$ with ORI. Oritavancin has a large volume of distribution $(\mathrm{Vd})(1.25 \mathrm{~L} / \mathrm{kg})$ indicating extensive tissue distribution, whereas DAL's smaller Vd $(0.11 \mathrm{~L} / \mathrm{kg})$ indicates it primarily remains in the plasma compartment $[9,17]$. Although we acknowledge the presence of these and other differences, the clinical impact is unclear as both drugs have been associated with successful outcomes in the treatment of complicated infections [12, 13, 14-16]. In addition, clinical scenarios exist where DAL may be preferred, such as for genitourinary sources as $<5 \%$ of ORI is recovered unchanged in the urine compared to $33 \%$ of DAL $[9,17]$.

Our study is not without limitations. All but one patient received ORI as secondary therapy and all bacteremic patients received the drug following clearance of blood cultures, so no determination may be made regarding the utility of this agent for primary therapy. Patients received varying regimens for similar indications and thus it is not possible to evaluate a specific regimen for a given indication. Finally, generalizability may be limited due to the single center design of the study.

\section{Conclusion}

In conclusion, we report our real-world experience using ORI in PWID primarily for treatment completion of complicated gram-positive infections. We acknowledge the delicate balance between antimicrobial stewardship efforts and financial considerations in providing a high cost, broad spectrum agent to ensure patients receive a safe, effective regimen that avoids extended hospitalizations and incomplete treatment courses. We believe ORI may be considered in patients who require prolonged therapy courses but are unable to receive OPAT, including PWID and patients experiencing homelessness. Randomized controlled studies should be conducted to determine optimal dosing regimens for off-label indications and to compare LAL therapy to standard of care. 


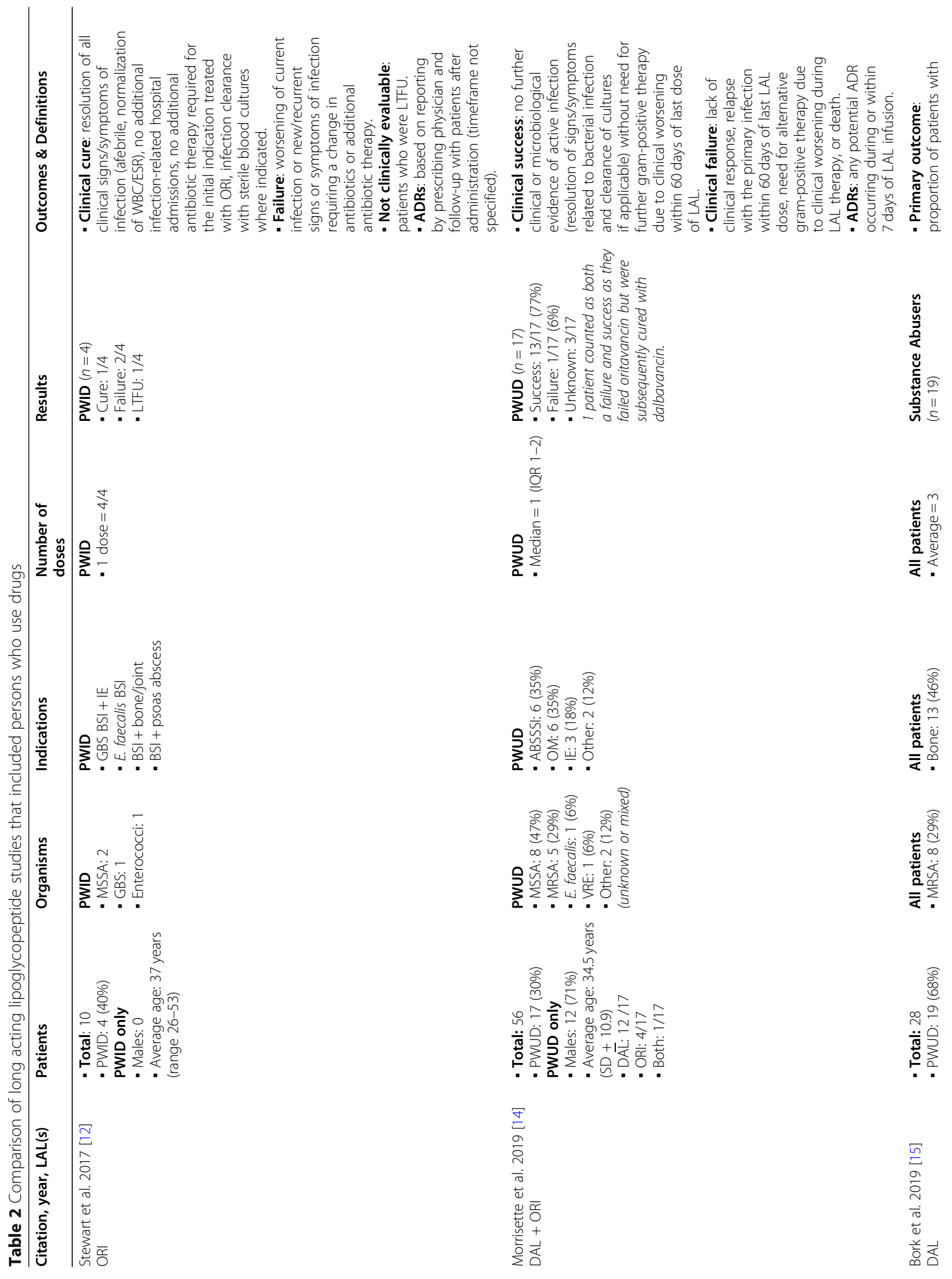




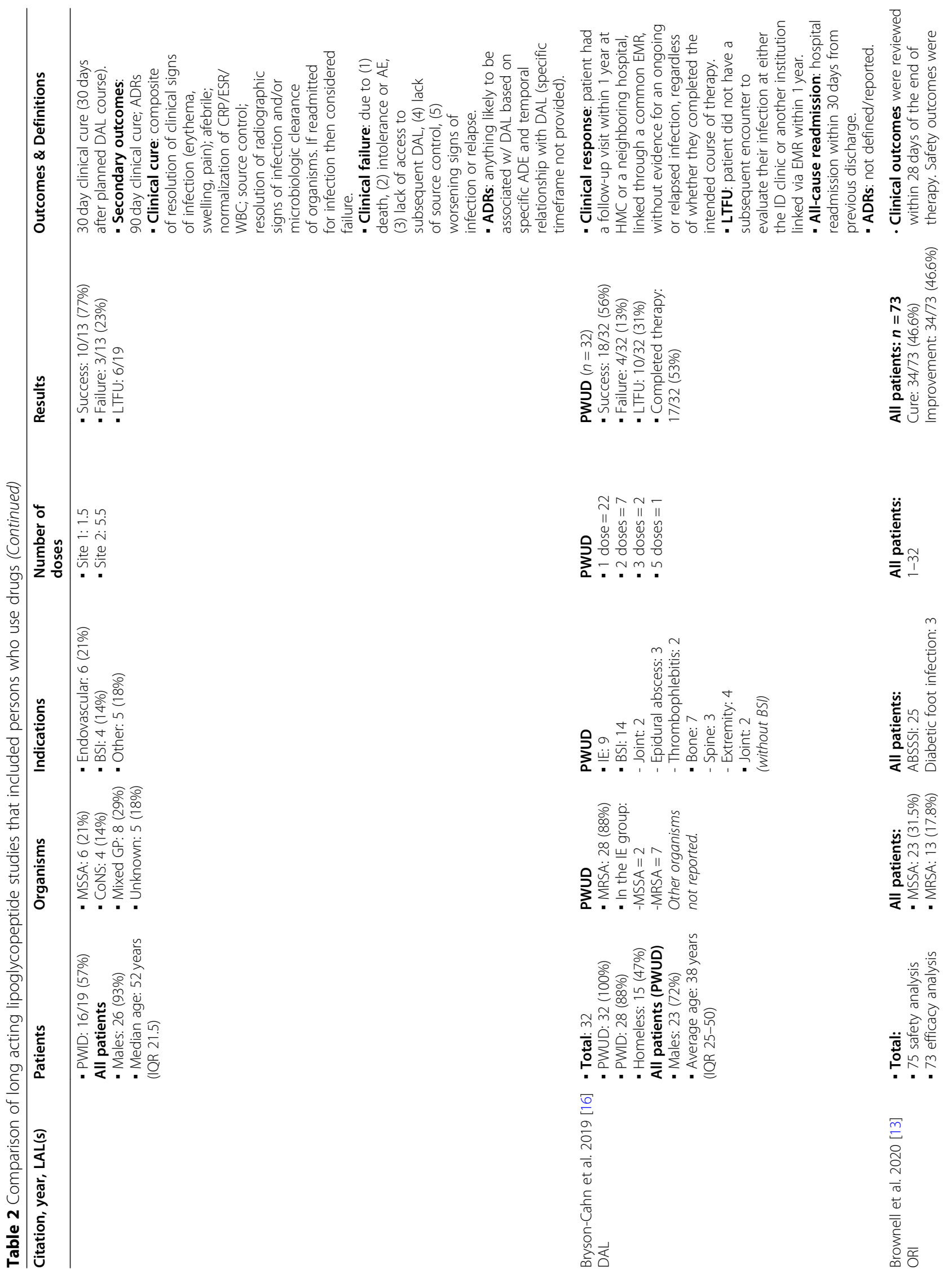




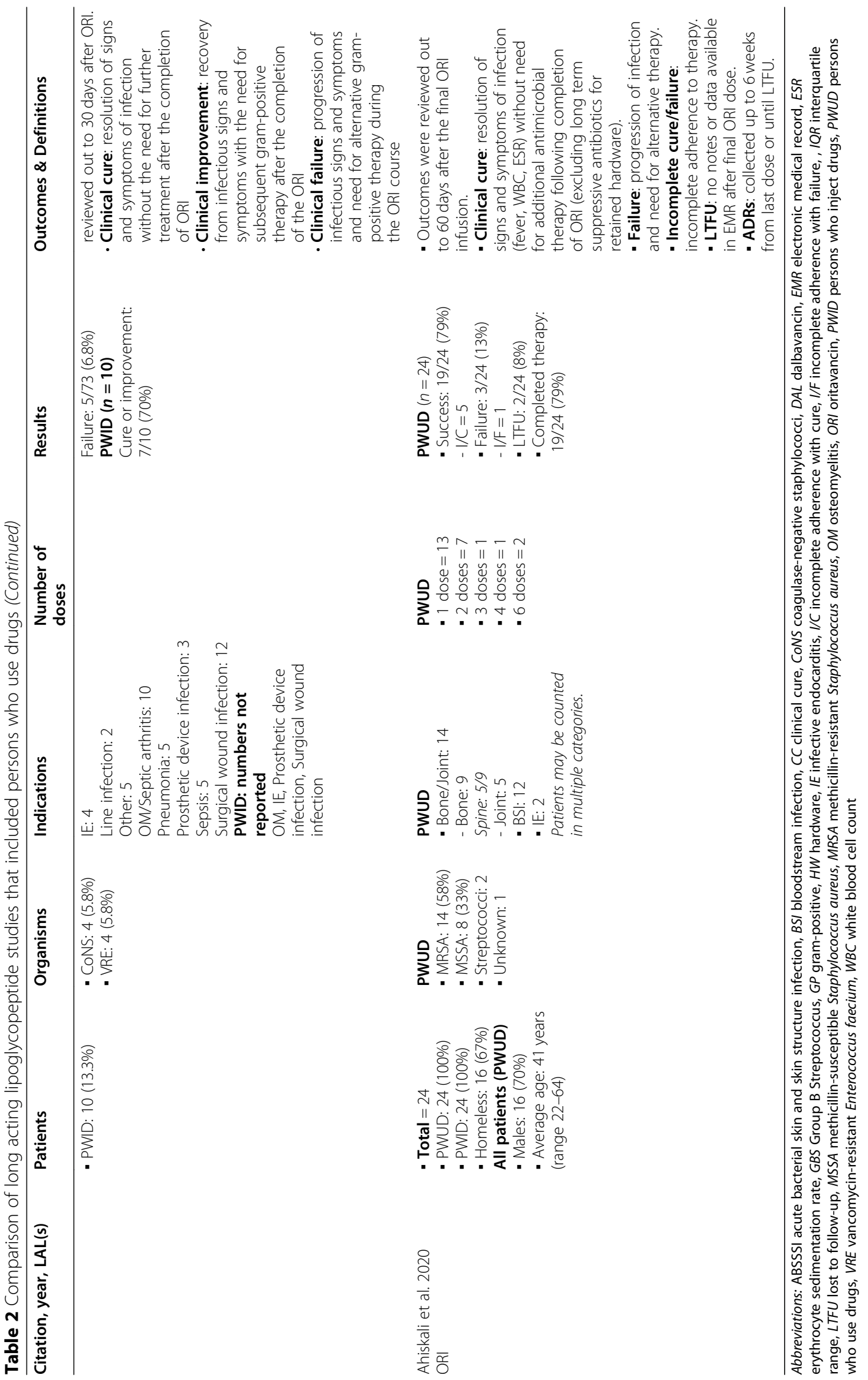




\section{Abbreviations}

PWID: Persons who inject drugs; MRSA: Methicillin resistant Staphylococcus aureus; IDU: Injection drug use; ABIs: Associated bacterial infections; OPAT: Outpatient parenteral antibiotic therapy; AMA: Against medical advice; SUD: Substance use disorder; ORI: Oritavancin; LAL: Long-acting lipoglycopeptide; MSSA: Methicillin susceptible Staphylococcus aureus; ABSSSI: Acute bacterial skin and skin structure infections; IV: Intravenous; DAL: Dalbavancin; HCMC: Hennepin County Medical Center; ID: Infectious diseases; I/C: Incomplete adherence/cure; I/F: Incomplete adherence/failure; ADRs: Adverse drug reactions; EMR: Electronic medical record; NADR PS: Naranjo Adverse Drug Reaction Probability Scale; BMI: Body mass index: ED: Emergency Department; MIC: Minimum inhibitory concentration; PICC: Peripherally inserted central catheter; TTE: Transthoracic echocardiography; PWUD: Persons who use drugs; t12/2: Half-life; Vd: Volume of distribution

\section{Acknowledgements}

Not applicable.

\section{Code availability}

Not applicable.

\section{Authors' contributions}

$A A$ and HR both made substantial contributions to the conception and design of the work, the analysis and interpretation of data and equally contributed to the development of the manuscript. AA and HR have both approved the submitted version and have agreed both to be personally accountable for their contributions and to ensure that questions related to the accuracy or integrity of any part of the work, even ones in which the author was not personally involved, are appropriately investigated, resolved, and the resolution documented in the literature.

\section{Funding}

No funding sources to report.

\section{Availability of data and materials}

All data analyzed during this study are included in this published article and may be found in Table 1 .

\section{Ethics approval and consent to participate}

The study was reviewed and approved by the Hennepin Healthcare Research Institute, study \# 19-4633.

\section{Consent for publication}

Not applicable.

\section{Competing interests}

On behalf of both authors, the corresponding author states that there is no conflict of interest.

\section{Author details}

'Department of Pharmacy, Hennepin Healthcare, 701 Park Avenue, Minneapolis, MN 55415, USA. ${ }^{2}$ Clinical Quality Improvement, Hennepin Healthcare, Orange 1.220-7, 701 Park Avenue, Minneapolis, MN 55415, USA.

\section{Received: 13 April 2020 Accepted: 8 October 2020}

\section{Published online: 28 October 2020}

\section{References}

1. Binswanger IA, Kral AH, Blumenthal RN, Rybold DJ, Edlin BR. High prevalence of abscesses and cellulitis among community-recruited injection drug users in San Francisco. Clin Infect Dis. 2000;30:579-81. https://doi.org/ 10.1086/313703.

2. Morrison A, Elliott L, Gruer L. Injecting-related harm and treatment-seeking behaviour among injecting drug users. Addiction. 1997;92:1349-52.

3. Phillips KT, Stein MD. Risk practices associated with bacterial infections among injection drug users in Denver, CO. Am J Alcohol Abuse. 2010;36(2): 92-7. https://doi.org/10.3109/00952991003592311.

4. Miller AC, Polgreen PM. Many opportunities to record, diagnose, or treat injection-drug related infections are missed: a population-based cohort study of inpatient and emergency department settings. Clin Infect Dis. 2019, 68(7):1166-75. https://doi.org/10.1093/cid/ciy632.

5. Song JY, Safaeian M, Strathdee SA, Vlahov D, Celentano DD. The prevalence of homelessness among injection drug users with and without HIV infection. J Urban Health. 2000;77(4):678-87. https://doi.org/10.1007/ BF02344031.

6. Linton SL, Celentano DD, Kirk GD, Mehta SH. The longitudinal association between homelessness, injection drug use, and injection-related risk behavior among persons with a history of injection drug use in Baltimore, MD. Drug Alcohol Depend. 2013;132(3):457-65. https://doi.org/10.1016/j. drugalcdep.2013.03.009.

7. Buehrle DJ, Shields RK, Shah N, Shoff C, Sheridan K. Risk factors associated with outpatient parenteral antibiotic therapy program failure among intravenous drug users. Open Forum Infect Dis. 2017;4:ofx102. https://doi. org/10.1093/ofid/ofx102.

8. Eaton EF, Matthew RE, Lane PS, et al. A 9-point risk assessment for patients who inject drugs and require intravenous antibiotics: focusing inpatient resources on patients at greatest risk of ongoing drug use. Clin Infect Dis. 2019;68(6):1041-3. https://doi.org/10.1093/cid/ciy722.

9. Product information. Orbactiv (Oritavancin) injection. Lincolnshire: Melinta Therapeutics; 2019.

10. Jones RN, Moeck G, Arhin FF, Dudley MN, Rhomberg PR, Mendes RE. Results from oritavancin resistance surveillance programs (2011 to 2014): clarification for using vancomycin as a surrogate to infer oritavancin susceptibility. Antimicrob Agents Chemother. 2016;60(5):3174-7. https://doi. org/10.1128/AAC.03029-15.

11. Naranjo CA, et al. A method of estimating the probability of adverse drug reactions. Clin Pharmacol Ther. 1981:30:239-45.

12. Stewart CL, Turner MS, Frens JJ, Snider CB, Smith JR. Real-world experience with oritavancin therapy in invasive gram-positive infections. Infect Dis Ther. 2017;6:277-89. https://doi.org/10.1007/s40121-017-0156-z.

13. Brownell LE, Adamsick ML, McCreary E, et al. Clinical outcomes and economic impact of oritavancin for gram-positive infections: a single academic medical center health system experience. Drugs - Real World Outcomes. 2020;7(suppl 1):S13-9.

14. Morrisette T, Miller MA, Montague BT, Barber GR, McQueen RB, Krsak M. Long-acting lipoglycopeptides: "lineless antibiotics" for serious infections in persons you use drugs. Open Forum Infect Dis. 2019;6(7):1-7. https://doi. org/10.1093/ofid/ofz274

15. Bork JT, Heil EL, Berry S, et al. Dalbavancin use in vulnerable patients receiving outpatient parenteral antibiotic therapy for invasive gram-positive infections. Infect Dis Ther. 2019;8:171-84. https://doi.org/10.1007/s40121019-0247-0.

16. Bryson-Cahn C, Beieler AM, Chan JD, Harrington RD, Dhanireddy S. Dalbavancin as secondary therapy for serious Staphylococcus aureus infections in a vulnerable patient population. Open Forum Infect Dis. 2019; 6(2):1-4. https://doi.org/10.1093/ofid/ofz028.

17. Product information. Dalvance (Dalbavancin) injection. Madison: Allergan USA, Inc.; 2018.

\section{Publisher's Note}

Springer Nature remains neutral with regard to jurisdictional claims in published maps and institutional affiliations.

\section{Ready to submit your research? Choose BMC and benefit from:}

- fast, convenient online submission

- thorough peer review by experienced researchers in your field

- rapid publication on acceptance

- support for research data, including large and complex data types

- gold Open Access which fosters wider collaboration and increased citations

- maximum visibility for your research: over $100 \mathrm{M}$ website views per year

At $\mathrm{BMC}$, research is always in progress.

Learn more biomedcentral.com/submissions 